\title{
Extent of Tibiofemoral Osteoarthritis Before Knee Arthroplasty: Multicenter Data from the Osteoarthritis Initiative
}

\author{
Daniel L. Riddle PhD, William A. Jiranek MD, \\ Robert S. Neff MD, Derek Whitaker MD, \\ Jason R. Hull MD
}

Received: 28 October 2011/Accepted: 16 March 2012/Published online: 27 March 2012

(C) The Association of Bone and Joint Surgeons (B) 2012

\begin{abstract}
Background Knee arthroplasty traditionally is recommended for persons with substantial disability and disabling pain attributable to moderate or severe osteoarthritis (OA). Pain and functional status after arthroplasty may be influenced by the extent of knee OA before surgery and recent evidence suggests persons with less severe knee OA before undergoing TKA have greater pain levels and worse function than persons with more severe knee OA. Questions/purposes We determined the proportion of patients undergoing knee arthroplasty who had less than moderate knee OA before surgery and who had either a radiographically normal medial or lateral joint space before surgery.

Methods One hundred sixteen persons in the Osteoarthritis Initiative underwent knee arthroplasty during a
\end{abstract}

Each author certifies that he or she, or a member of his or her immediate family, has no commercial associations (eg, consultancies, stock ownership, equity interest, patent/licensing arrangements, etc) that might pose a conflict of interest in connection with the submitted article.

All ICMJE Conflict of Interest Forms for authors and Clinical Orthopaedics and Related Research editors and board members are on file with the publication and can be viewed on request. Each author certifies that his or her institution approved the reporting of this report, that all investigations were conducted in conformity with ethical principles of research, and that informed consent for participation in the study was obtained.

\section{L. Riddle ( $\square)$}

Department of Physical Therapy, Virginia Commonwealth University, P.O. Box 980224, Richmond, VA 23298, USA

e-mail: dlriddle@vcu.edu

D. L. Riddle, W. A. Jiranek, R. S. Neff, D. Whitaker, J. R. Hull Department of Orthopaedic Surgery, Virginia Commonwealth University, Richmond, VA, USA
3 -year period. Ninety-seven of the 116 patients (84\%) had radiographs available less than 1 year before surgery and were included. We used $Z$-tests to determine whether the proportion of patients with a modified Kellgren-Lawrence (KL) grade of 3 or higher differed from literature-based estimates. In addition, we described the proportion of patients with medial and lateral joint space narrowing.

Results The proportion of patients with a modified KL grade of 3 or higher was 0.81 (95\% CI, 0.73-0.89) and was less than the 0.95 estimated population proportion. Of the patients who underwent knee arthroplasty, 85\% (82 of 97 knee arthroplasties) had at least one tibiofemoral joint compartment that had no joint space narrowing. One in six patients with OA who underwent knee arthroplasty had a KL grade of 2 or lower.

Conclusions Variation in the extent of tibiofemoral OA in patients preparing for joint arthroplasty is greater than previously described. A greater percentage of patients undergoing knee arthroplasty may be at risk for increased pain and poorer function than previously assumed after surgery because of less severe knee OA before surgery.

Level of Evidence Level I, diagnostic study. See Guidelines for Authors for a complete description of levels of evidence.

\section{Introduction}

The decision to recommend knee arthroplasty is a complex one that involves simultaneous consideration of multiple factors, including but not limited to symptom intensity and duration, functional loss, osteoarthritis (OA) severity, ligamentous and patellofemoral joint integrity, and response to previous therapies. Judgments of the extent and type of tibiofemoral OA play an important role when advising 
patients about risks and benefits of joint arthroplasty. As reported in a NIH-sponsored consensus meeting [18] and corroborated by others $[7,9]$, primary TKA is most commonly performed for knee failure caused by OA. However, there is controversy regarding specific indications for knee arthroplasty and the literature suggests substantial variation in indications [7, 10, 11]. For example, there is a lack of consensus regarding duration and extent of pain or functional loss necessary to indicate a need for TKA $[7,11]$. Although the patellofemoral joint is important when considering a patient's candidacy for TKA, the tibiofemoral joint is likely given highest priority because of its primary weightbearing function. Recent evidence suggests persons with less severe knee OA before undergoing TKA have greater pain levels and worse function than persons with more severe knee OA $[8,23]$.

Lateral and medial compartments of the tibiofemoral joint are vulnerable to $\mathrm{OA}$ and are influenced by different racial, bony alignment, movement, and injury-related risk factors [28]. Assessing the lateral and medial compartments of the tibiofemoral joint also is clinically important because surgical options can vary, in part, depending on the OA status of these compartments. The extent to which the medial and lateral joint spaces are affected by OA before surgery is unclear. However, because implant decisions are driven at least in part by the extent of medial and lateral joint space disease it is clinically important to understand the extent of medial versus lateral joint space narrowing. Plain radiographs are commonly used by orthopaedic surgeons when planning surgical treatment for patients with knee OA [12]. A Kellgren-Lawrence (KL) grade [14] of 2 is considered indicative of OA, primarily because of the presence of osteophytes. When tibiofemoral joint space narrowing is definite, a grade of 3 is given and a grade of 4 is used for marked narrowing. A KL grade of 3 or 4 traditionally has been suggested as an indicator for TKA consideration, presumably because a grade of either 3 or 4 reflects definitive joint space narrowing, the impairment targeted by the implant.

Authors of three studies conducted in Europe $[3,13]$ and Asia [15] reported KL grades for persons scheduled for knee arthroplasty and all reported the proportion of knees with a KL grade of 3 or 4 exceeded $95 \%$. Authors of one recently published US-based study [25] reported $83.2 \%$ of knees had a KL grade of 3 or 4 before knee arthroplasty, but the time between radiographic assessment and subsequent surgery was not reported and could have been as much as 30 months. Another study [26] examined the extent of medial versus lateral tibiofemoral joint space narrowing in patients selected for TKA, but standardized methods for obtaining or reading radiographs were not used. Given that knee arthroplasty generally is recommended for persons with moderate or severe knee OA, we presumed approximately $95 \%$ of persons would have a modified KL grade of 3 or higher in the involved knee within 1 year of surgery, an estimate consistent with the literature [3, 13, 15]. Further, given surgical treatment decisions are driven at least to some extent by the pattern of tibiofemoral joint space narrowing, it is important to understand not only the extent, but also the pattern of joint space narrowing.

The aims of our study were to (1) determine the severity of tibiofemoral arthritis less than 1 year before knee arthroplasty and (2) describe the proportion of persons with a radiographically normal medial or lateral tibiofemoral joint space before arthroplasty.

\section{Patients and Methods}

The Osteoarthritis Initiative (OAI) is a publicly and privately funded prospective 4-year longitudinal cohort study of 4796 persons aged 45 to 79 years with knee OA or at risk for knee OA. Subjects received no treatment as part of the study nor were they solicited for treatment by the investigators. Patients assigned to the incidence and progression subcohorts who subsequently underwent knee arthroplasty were included in the study. The incidence subcohort comprised 3285 persons who either had knee pain without OA or no knee pain but with OA or were at risk for having knee OA develop. The progression subcohort had 1389 persons with symptomatic knee OA in one or both knees. Symptomatic knee OA was defined in the OAI as (1) a self-report of pain, aching, or stiffness in or around one or both knees on most days for at least 1 month during the past 12 months and (2) radiographic tibiofemoral knee OA defined as Osteoarthritis Research Society International (OARSI) Grades 1 to $3[1,2]$ or the approximate equivalent, modified KL grades of 2 or higher $[1,2]$. Subjects were recruited from the communities near the following institutions: (1) University of Maryland in Baltimore, MD, USA; (2) The Ohio State University in Columbus, OH, USA; (3) University of Pittsburgh in Pittsburgh, PA, USA; and (4) Memorial Hospital of Rhode Island, in Pawtucket, RI, USA. Key exclusion criteria for entry into the study were (1) the presence of rheumatoid arthritis, (2) bilateral knee arthroplasties before study entry or preexisting plans to undergo bilateral (not unilateral) knee arthroplasties in the next 3 years, (3) bilateral endstage (modified KL Grade 4) knee OA, (4) use of ambulatory aids other than one straight cane for greater than $50 \%$ of the time, (5) comorbid conditions that might interfere with 4-year participation, and (6) likelihood of not residing in the clinic area for at least 3 years. In addition, because the study measured MRI-based changes, men weighing greater than $130 \mathrm{~kg}$ and women weighing greater 
than $114 \mathrm{~kg}$ (1595 persons) were excluded because they were unable to undergo 3.0-T MRI. The study was approved by the institutional review board of the OAI Coordinating Center at the University of California, San Francisco (San Francisco, CA, USA).

During the course of 3 years of followup, 116 persons had knee arthroplasties and this was the sample we included in the study (Table 1). A total of 27 persons had knee arthroplasties during Year 1, 38 during Year 2, and 51 during Year 3 of the study. Nineteen of the 116 patients had more than one knee arthroplasty, with 13 undergoing bilateral arthroplasties in the same year and six with either repeated unilateral arthroplasties in different years or contralateral arthroplasties in different years. We selected only one knee per patient. For the patients with bilateral arthroplasties, we randomly selected either the right or left knee. For the unilateral arthroplasties, we selected the first surgical episode.

Radiographs obtained less than 1 year before surgery were available for 97 patients. Radiographic examinations were obtained an average of 163 days $(\mathrm{SD}=101.2)$ before surgery. Of the 97 patients, one had a unicompartmental arthroplasty and the remaining 96 underwent TKAs. We compared the demographic characteristics of the persons with $(\mathrm{n}=97)$ and without $(\mathrm{n}=19)$ radiographic data available within 1 year of surgery and no differences in age, sex, or comorbidity score were found ( $\mathrm{p}$ values of t-tests for age and comorbidity $=0.77$ and 0.70 respectively and chi-square for gender $=0.60$ ).

We conducted a power analysis to assess the adequacy of our sample size. With a one-group chi-square test and a two-sided significance level set at $\alpha=0.05$ and a sample size of 90 , our study had a power of $92 \%$ to detect a difference of 0.10 in the prevalence of KL grade of 3 or higher between the null hypothesis population (0.95) and the preknee arthroplasty OAI sample $(\leq 0.85)$. We reasoned, if the proportion of patients with KL grades of 3 or greater was at least 0.10 lower than previously published estimates, the clinical implications potentially would be important; for example, if $15 \%$ of persons undergoing TKAs had KL grades less than 3, this would indicate a substantial number of patients potentially would be at risk for worse pain and function relative to persons with KL grades of 3 or greater $[8,23]$.

OAI investigators used a $20^{\circ}$ fixed-flexion bilateral weightbearing posteroanterior radiographic approach to optimally capture the tibiofemoral joint space $[6,20]$. Radiographs were obtained using a SynaFlexer ${ }^{\mathrm{TM}}$ frame (Synarc, San Francisco, CA, USA) to reproducibly position the subject's knees and feet and to equally distribute body weight while the $\mathrm{x}$-ray beam was angled caudally at $10^{\circ}$ from a preset distance [16]. The radiologic staff at the institutions underwent extensive training to enhance consistency. Lateral or skyline view radiographs were not obtained as part of the OAI study.

Radiographs were obtained at baseline and at the 1-year and 2-year followups. OAI site investigators assessed baseline radiographs and used modified KL and OARSI grading scales to quantify the extent of OA of both knees in each radiograph (Table 1). At followups, there were no radiographic readings provided for the sample in the public use datasets. OAI researchers used an extensive training

Table 1. Demographic and clinical data for the 116 persons undergoing knee arthroplasties

\begin{tabular}{llc}
\hline Variable & Value* & $\begin{array}{c}\text { Missing data } \\
\text { (number of knees) }\end{array}$ \\
\hline Age (years) & $65.03(8.30,45,78)$ & 0 \\
Female & $68(58.6 \%)$ & 1 \\
Race & & $2(1.7 \%)$ \\
Other nonwhite & $92(80.0 \%)$ & 0 \\
White & $19(16.5 \%)$ & 10 \\
Black or African American & $2(1.7 \%)$ & 19 \\
Asian & $3(2.6 \%)$ & \\
Hispanic or Latino & $0.51(0.86,0,3)$ \\
Comorbidity score (points) & $30.15(5.11,19.2,43)$ & \\
BMI & $163(101.2,2,378)$ \\
Time from radiography to TKA (days) & & 12 \\
Self-reported functional status (points) & $57.02(7.38,34.3,72.84)$ & 6 \\
SF-12 mental summary score & $37.38(10.2,14.41,57.49)$ & \\
SF-12 physical summary score & $39.2(18.09,0,75)$ & \\
KOOS quality of life & &
\end{tabular}

* Values are expressed as mean, with SD, minimum, and maximum in parentheses, or as number of knees; KOOS = Knee Injury and Osteoarthritis Outcome Score. 
approach to maximize reliability and validity of baseline radiograph readings. Site investigators were experienced rheumatologists and all received standardization training in the KL and OARSI grading scales using teleconferences and a Web-based program. Before the study, each site investigator read 30 knee radiographs and provided modified KL and OARSI grades. They then were given feedback and the correct answers with annotated images and interpretive comments by an experienced musculoskeletal radiologist. During the validation process, investigators read different sets of 30 knee radiographs and their results were compared against the gold standard readings made by an experienced musculoskeletal radiologist. All site investigators had to pass the validation test before reading OAI radiographs.

Because OAI investigators only provided modified KL and medial and lateral joint space readings for baseline data and not for the followup radiographs, we recruited four orthopaedic surgeons (WAJ, RSN, DW, JRH) to make $\mathrm{KL}$, modified KL, and OARSI readings on a total of 200 baseline and 1- and 2-year followup radiographs of patients who subsequently had knee arthroplasties. Surgeons were blinded to TKA status or involved side. Surgeons also were unaware of the baseline OAI KL or OARSI readings for each radiograph. All surgeons were provided with the $\mathrm{KL}$, modified KL, and OARSI scales and radiographic examples [1]. The KL and modified KL systems are semiquantitative and define the extent of knee OA by the presence and severity of osteophytes and tibiofemoral joint space narrowing. The OARSI system defines the extent of either medial or lateral tibiofemoral joint space narrowing based on a percentage estimate relative to normal. The surgeons practiced individually on 10 radiographs until they were prepared to use the scales to grade the radiographs. We used the weighted kappa to determine the extent of agreement between each surgeon's readings of the baseline radiographs and the readings made by the OAI site investigators. The weighted kappa is the chancecorrected agreement coefficient when using ordinal scales and when differences in agreement become more serious as disagreements become greater [17, 22]. One surgeon (JRH) read 225 radiographs from the OAI dataset and had weighted kappas for interobserver variability of 0.67 to 0.84 when compared with publically available baseline radiographic readings by OAI investigators of the same subjects (Table 2). Weighted kappa coefficients are commonly used agreement indices that account for chance agreement in ordinal scale measures like the radiographic assessments conducted in this study [17]. Because of strong concordance with OAI readings, we considered this surgeon's radiographic readings to be valid and we used the surgeon's data from baseline and followup readings to examine the extent of medial and lateral joint space narrowing and KL grades.

We determined differences in the proportion of patients with modified KL grades of 3 or higher, indicating at least moderate knee OA, between our sample of persons with TKAs and a fixed proportion of 0.95 based on evidencebased estimates [3, 13, 15] using the Z-test. Descriptive statistics were used to summarize the extent of medial versus lateral joint space narrowing. All data were analyzed with SPSS ${ }^{\circledR}$ Statistics (Version 19; IBM, Armonk, NY, USA).

\section{Results}

The proportion of patients with a modified KL grade of 3 or higher was 0.81 (95\% CI, 0.73, 0.89) (Table 3). This proportion was lower $(Z=6.36 ; \mathrm{p}<0.001)$ than the 0.95 estimated population proportion.

A total of 23 (24\%) of the 97 surgical knees had no joint space narrowing in the medial compartment and $64(66 \%)$ had no tibiofemoral narrowing in the lateral compartment (Table 4). Stated another way, 82 of 97 patients had a presurgical knee $(85 \%$; 95\% CI, 78\%, 92\%) that had at least one tibiofemoral joint compartment with no joint space narrowing. A total of $5 \%$ of surgical knees had no

Table 3. Distribution of Kellgren-Lawrence grades for $96^{*}$ patients who had TKA within 1 year of radiographic examination

\begin{tabular}{lccc}
\hline $\begin{array}{l}\text { Kellgren-Lawrence } \\
\text { grade }\end{array}$ & $\begin{array}{l}\text { Frequency } \\
\text { (number of knees) }\end{array}$ & Percentage & $\begin{array}{l}\text { Cumulative } \\
\text { percentage }\end{array}$ \\
\hline 0 & 1 & 1.0 & 1.0 \\
1 & 8 & 8.3 & 9.4 \\
2 & 9 & 9.4 & 18.8 \\
3 & 36 & 37.5 & 56.3 \\
4 & 42 & 43.8 & 100 \\
\hline
\end{tabular}

* Kellgren-Lawrence data were missing for one of the 97 patients who had radiographic data.

Table 2. Extent of agreement between orthopaedic surgeon and OAI site investigators using bilateral baseline OAI knee radiographic data

\begin{tabular}{|c|c|c|c|c|}
\hline Measurement & Number & $\kappa_{\mathrm{w}}$ & $95 \%$ CI for $\kappa_{\mathrm{w}}$ & $\kappa_{\mathrm{w}(\max )}$ \\
\hline Medial joint space narrowing & 225 & 0.73 & $0.60,0.85$ & 0.84 \\
\hline Lateral joint space narrowing & 225 & 0.67 & $0.54,0.80$ & 0.76 \\
\hline Modified Kellgren-Lawrence grade & 224 & 0.84 & $0.76,0.91$ & 0.94 \\
\hline
\end{tabular}

$\mathrm{OAI}=$ Osteoarthritis Initiative; $\kappa_{\mathrm{w}}=$ weighted kappa. 
Table 4. Distribution of joint space narrowing for 97 patients

\begin{tabular}{llllrl}
\hline Joint space & \multicolumn{2}{l}{ Lateral } & & & \\
\cline { 2 - 6 } narrowing & 0 & 1 & 2 & 3 & Total \\
\hline Medial & 5 & 3 & 2 & 13 & 23 \\
0 & 5 & 0 & 4 & 4 & 13 \\
1 & 11 & 1 & 1 & 1 & 14 \\
2 & 43 & 3 & 0 & 1 & 47 \\
3 & 64 & 7 & 7 & 19 & 97 \\
Total & & & & & \\
\hline
\end{tabular}

joint space narrowing in either the medial or lateral compartment.

\section{Discussion}

Knee arthroplasty traditionally is recommended for persons with substantial disability and disabling pain attributable to moderate or severe OA. Pain and functional status after arthroplasty may be influenced by the extent of knee OA before surgery. Our study determined the extent and type of OA in patients less than 1 year before knee arthroplasty. Specifically, we determined the proportion of patients who had KL grades less than 3 before their TKA. Less severe knee OA before surgery reportedly is associated with greater postoperative pain and worse functional status [8, 23]. Surgeons should be aware that less than a moderate amount of knee OA before TKA may place a patient at risk for poor outcome. In addition, decisions related to the use of total versus unicompartmental surgery are driven, at least to some extent, by the extent of compartmental OA. We therefore described the proportion of patients who had no radiographic evidence of medial or lateral joint space narrowing.

Our study has several limitations that warrant consideration. First, our sample size is relatively small and overall, the knee OA disease spectrum of the population of persons in the OAI generally was skewed toward mild to moderate disease. However, to our knowledge, the OAI study is the literature's largest and most diverse communitybased sample of persons with symptomatic knee OA studied during a 3-year period. Despite the small sample, the statistical estimates were robust and the data were collected in four geographically diverse regions with approximately equal representation among the sites. We cannot say, however, that these data reflect those for the entire United States or other countries. Second, all radiographic data were collected, on average, 163 days before knee arthroplasty. It is possible that additional joint space narrowing occurred between the radiograph and the surgery. However, yearly changes in joint space width average approximately 0.1 to $0.2 \mathrm{~mm} /$ year $[4,12]$. Given that the time between the radiograph and arthroplasty was much less than 1 year for most patients, the very small yearly changes that typically occur [4, 12] suggest the time between radiographic examination and surgery had little effect on OA status. Third, we had no data regarding the status of the patellofemoral joints because radiographs of the patellofemoral joints were not obtained. Isolated patellofemoral arthroplasty, however, is a rare procedure. In Norway in 2009 , only $0.4 \%$ of all knee arthroplasties were isolated patellofemoral arthroplasties [19]. Although all but one patient with complete data in our study underwent TKA, some patients who underwent arthroplasties may have had extensive patellofemoral OA that we were unable to quantify. Fourth, the extent of variation in criteria used to recommend knee arthroplasty are well documented and tibiofemoral OA is only one of several criteria [7, 27]. Because we did not have data for all variables used to make surgical decisions (eg, patellofemoral status or osteonecrosis) and because consensus regarding who should receive knee arthroplasty has not been established in the United States, we cannot judge the appropriateness of surgical decisions made in our sample. Fifth, we relied on data from one surgeon whose radiographic measures were highly concordant with OAI investigators' data. Even with a high concordance, there were likely some radiographs that were read incorrectly by either the surgeon or the OAI investigators that contributed some error to our estimates. Sixth, a radiographic classification such as OARSI or KL cannot account for some bony or soft tissue changes, which can cause substantial symptoms in the absence of moderate or severe arthritis, including but not limited to (1) partial chondral loss, (2) subchondral edema, (3) joint surface subluxation, and (4) ligamentous insufficiency. In addition, the OAI did not provide stress radiography or diagnostic arthroscopy data. Soft tissue or bony disorders can contribute to pain and limited knee function and may lead patients to consider joint arthroplasty. Finally, the OAI had several exclusionary criteria including rheumatoid arthritis, a high body weight (eg, $130 \mathrm{~kg}$ or higher for men), and less than end-stage OA of both knees. These criteria limit the generalizability of our findings. In addition, persons in the OAI may be different from the US population because they were willing to volunteer for a long-term study of knee OA. Finally, the types of prostheses being implanted likely varied across sites and these data were not available in the OAI dataset.

Contrary to our expectation, we found the proportion of patients with a KL grade of 3 or greater planning for TKA was less than that expected from literature-based estimates, with $81 \%$ of knees in our sample having a KL grade of 3 or higher an average of 163 days before surgery. A NIH consensus panel described the most common indication as knee failure [18 ], so presumably the majority of patients 
undergoing the procedure for OA have KL grades of 3 or higher, indicating, at a minimum, definite tibiofemoral joint space narrowing. We found three studies, all conducted in Europe or Asia, which reported KL grades for knees before undergoing knee arthroplasties. All studies reported greater than $95 \%$ of patients had KL grades of 3 or higher $[3,13,15]$. A prevalence of KL grades of 3 or higher of $81 \%$ in our study is appreciably below the estimates from these three studies. A recently published paper by Wise et al. [25] indicated $83 \%$ of 202 knees undergoing TKAs in a multicenter longitudinal US study of knee OA had KL grades of 3 or higher before surgery, but radiographic data were collected up to 30 months before surgery for some patients. Given this limitation, our estimates were reasonably close to theirs. Taken together, these data represent approximately 300 TKAs conducted by communityand university-based surgeons in seven locations in the United States. Our data and the data by Wise et al. [25] suggest, for approximately one in six patients in the United States who approximate the OAI inclusionary and exclusionary criteria, TKA is being performed before the development of moderate or severe knee OA.

The percentage of patients with no evidence of joint space narrowing in at least one compartment was $85 \%$. This very high rate of unicompartmental disease suggests unicompartmental arthroplasty may be underutilized [5, 24]. Riddle et al. [21] reported unicompartmental knee arthroplasties account for less that $8 \%$ of all knee arthroplasties in the United States. More research is needed to confirm this high rate of unicompartmental disease and to further determine the extent to which these patients may be candidates for unicompartmental arthroplasty.

Although tibiofemoral OA status is only one of several criteria orthopaedic surgeons use to make surgical decisions, our study suggests variation in the extent of tibiofemoral OA in patients preparing for joint arthroplasty is greater than previously described. Similar to the recently published data by Wise et al. [25], approximately $19 \%$ of patients in our sample with KL grades of 2 or lower underwent knee arthroplasties. These data suggest a substantial number of patients are undergoing knee arthroplasty earlier in the disease process than has been previously reported. Evidence suggests these types of patients may be at risk for poorer pain and functional status outcomes [8, 23]. Additional research is needed to confirm these findings and to understand the reasons for the variation in the extent of OA before knee arthroplasty. In addition, the extent of medial versus lateral joint space narrowing is extremely variable, with $85 \%$ of knees having either a normal medial or a normal lateral joint space. Future research should more thoroughly examine additional attributes of patients being considered for knee arthroplasty to determine whether unicompartmental arthroplasty may be a reasonable option.

\section{References}

1. Altman RD, Gold GE. Atlas of individual radiographic features in osteoarthritis, revised. Osteoarthritis Cartilage. 2007;15(suppl A): A1-A56.

2. Altman RD, Hochberg M, Murphy WA Jr, Wolfe F, Lequesne M. Atlas of individual radiographic features in osteoarthritis. Osteoarthritis Cartilage. 1995;3(suppl A):3-70.

3. Barker K, Lamb SE, Toye F, Jackson S, Barrington S. Association between radiographic joint space narrowing, function, pain and muscle power in severe osteoarthritis of the knee. Clin Rehabil. 2004;18:793-800.

4. Benichou OD, Hunter DJ, Nelson DR, Guermazi A, Eckstein F, Kwoh K, Myers SL, Wirth W, Duryea J; Osteoarthritis Initiative Investigators. One-year change in radiographic joint space width in patients with unilateral joint space narrowing: data from the Osteoarthritis Initiative. Arthritis Care Res (Hoboken). 2010;62: 924-931.

5. Borus T, Thornhill T. Unicompartmental knee arthroplasty. J Am Acad Orthop Surg. 2008;16:9-18.

6. Brandt KD, Mazzuca SA, Conrozier T, Dacre JE, Peterfy CG, Provvedini D, Ravaud P, Taccoen A, Vignon E. Which is the best radiographic protocol for a clinical trial of a structure modifying drug in patients with knee osteoarthritis? J Rheumatol. 2002;29: $1308-1320$.

7. Cross WW 3rd, Saleh KJ, Wilt TJ, Kane RL. Agreement about indications for total knee arthroplasty. Clin Orthop Relat Res. 2006;446:34-39.

8. Cushnaghan J, Bennett J, Reading I, Croft P, Byng P, Cox K, Dieppe P, Coggon D, Cooper C. Long-term outcome following total knee arthroplasty: a controlled longitudinal study. Ann Rheum Dis. 2009;68:642-647.

9. Ethgen O, Bruyere O, Richy F, Dardennes C, Reginster JY. Health-related quality of life in total hip and total knee arthroplasty: a qualitative and systematic review of the literature. J Bone Joint Surg Am. 2004;86:963-974.

10. Gossec L, Paternotte S, Bingham CO 3rd, Clegg DO, Coste P, Conaghan PG, Davis AM, Giacovelli G, Gunther KP, Hawker G, Hochberg MC, Jordan JM, Katz JN, Kloppenburg M, Lanzarotti A, Lim K, Lohmander LS, Mahomed NN, Maillefert JF, Manno RL, March LM, Mazzuca SA, Pavelka K, Punzi L, Roos EM, Rovati LC, Shi H, Singh JA, Suarez-Almazor ME, Tajana-Messi E, Dougados M; OARSI-OMERACT Task Force Total Articular Replacement as Outcome Measure in OA. OARSI/OMERACT initiative to define states of severity and indication for joint replacement in hip and knee osteoarthritis. An OMERACT 10 Special Interest Group. J Rheumatol. 2011;38:1765-1769.

11. Gossec L, Paternotte S, Maillefert JF, Combescure C, Conaghan PG, Davis AM, Gunther KP, Hawker G, Hochberg M, Katz JN, Kloppenburg M, Lim K, Lohmander LS, Mahomed NN, March L, Pavelka K, Punzi L, Roos EM, Sanchez-Riera L, Singh JA, Suarez-Almazor ME, Dougados M; OARSI-OMERACT Task Force "total articular replacement as outcome measure in OA". The role of pain and functional impairment in the decision to recommend total joint replacement in hip and knee osteoarthritis: an international cross-sectional study of 1909 patients. Report of the OARSI-OMERACT Task Force on total joint replacement. Osteoarthritis Cartilage. 2011;19:147-154.

12. Guermazi A, Hunter DJ, Roemer FW. Plain radiography and magnetic resonance imaging diagnostics in osteoarthritis: validated staging and scoring. J Bone Joint Surg Am. 2009;91(suppl 1): 54-62.

13. Kauppila AM, Kyllonen E, Mikkonen P, Ohtonen P, Laine V, Siira P, Niinimaki J, Arokoski JP. Disability in end-stage knee osteoarthritis. Disabil Rehabil. 2009;31:370-380. 
14. Kellgren JH, Lawrence JS. Radiological assessment of osteoarthrosis. Ann Rheum Dis. 1957;16:494-502.

15. Kim HA, Kim S, Seo YI, Choi HJ, Seong SC, Song YW, Hunter D, Zhang Y. The epidemiology of total knee replacement in South Korea: national registry data. Rheumatology (Oxford). 2008;47:88-91.

16. Kothari M, Guermazi A, von Ingersleben G, Miaux Y, Sieffert M, Block JE, Stevens R, Peterfy CG. Fixed-flexion radiography of the knee provides reproducible joint space width measurements in osteoarthritis. Eur Radiol. 2004;14:1568-1573.

17. Landis JR, Koch GG. The measurement of observer agreement for categorical data. Biometrics. 1977;33:159-174.

18. NIH Consensus Panel. NIH Consensus Statement on total knee replacement December 8-10, 2003. J Bone Joint Surg Am. 2004; 86:1328-1335.

19. Norwegian Arthroplasty Register. Annual Report June 2010. Available at: http://nrlweb.ihelse.net/eng/Report_2010.pdf. Accessed January 4, 2012.

20. Peterfy C, Li J, Zaim S, Duryea J, Lynch J, Miaux Y, Yu W, Genant HK. Comparison of fixed-flexion positioning with fluoroscopic semi-flexed positioning for quantifying radiographic joint-space width in the knee: test-retest reproducibility. Skeletal Radiol. 2003;32:128-132.

21. Riddle DL, Jiranek WA, McGlynn FJ. Yearly incidence of unicompartmental knee arthroplasty in the United States. J Arthroplasty. 2008;23:408-412.
22. Sim J, Wright CC. The kappa statistic in reliability studies: use, interpretation, and sample size requirements. Phys Ther. 2005; 85:257-268.

23. Valdes AM, Doherty SA, Zhang W, Muir KR, Maciewicz RA, Doherty $M$. Inverse relationship between preoperative radiographic severity and postoperative pain in patients with osteoarthritis who have undergone total joint arthroplasty. Semin Arthritis Rheum. 2012;41:568-575.

24. Willis-Owen CA, Brust K, Alsop H, Miraldo M, Cobb JP. Unicondylar knee arthroplasty in the UK National Health Service: an analysis of candidacy, outcome and cost efficacy. Knee. 2009;16:473-478.

25. Wise BL, Felson DT, Clancy M, Niu J, Neogi T, Lane NE, Hietpas J, Curtis JR, Bradley LA, Torner JC, Zhang Y. Consistency of knee pain and risk of knee replacement: the Multicenter Osteoarthritis Study. J Rheumatol. 2011;38:13901395.

26. Woolson ST, Shu B, Giori NJ. Incidence of radiographic unicompartmental arthritis in patients undergoing knee arthroplasty. Orthopedics. 2010;33:798.

27. Wright JG, Coyte P, Hawker G, Bombardier C, Cooke D, Heck D, Dittus R, Freund D. Variation in orthopedic surgeons' perceptions of the indications for and outcomes of knee replacement. CMAJ. 1995;152:687-697.

28. Zhang Y, Jordan JM. Epidemiology of osteoarthritis. Rheum Dis Clin North Am. 2008;34:515-529. 